\title{
RASGOS MORFOLÓGICOS DE LOS VOLCANES RECIENTES DE MONTAÑAS NEGRAS EN SANTIAGO DEL TEIDE (ISLA DE TENERIFE)
}

\author{
Francisco Javier DÓNIZ PÁEZ \\ Departamento de Geografía. Universidad de La Laguna
}

\section{INTRODUCCIÓN.}

Los volcanes de Montañas Negras en Santiago del Teide constituyen un conjunto eruptivo de naturaleza basáltica formado por cuatro aparatos volcánicos de carácter simple y tres derrames lávicos asociados. Se localizan en el área de cumbres de la estructura volcánica compleja de la Dorsal de Bilma, a una altitud que oscila entre los 1.300-1.350 metros aproximadamente. Están ubicados en un estrecho pasillo intravolcánico enmarcado por edificios de carácter monogénico, al norte Montaña de la Cruz y del Estrecho con 1.530 y 1.461 metros, respectivamente, y al sur Montaña Poleos con 1.488 metros de altitud, e inscritos en un islote que se configura a partir de los derrames lávicos de la erupción de 1909.

La instalación de este conjunto eruptivo de edad reciente provocó una remodelación puntual de una morfoestructura de mayor envergadura como es la Dorsal de Bilma. La discontinuidad espaciotemporal del fenómeno eruptivo, caracteriza, en gran medida, la interferencia entre los propios procesos de construcción y a su vez, entre los de construcción y erosión, dando lugar a la imbricación de las formas de relieve que constituyen el paisaje propio de las dorsales del Archipiélago (DÓNIZ ET AL, 1999: 87). De este modo, los procesos dominantes son los relacionados con la actividad volcánica manifestada a través de la presencia de formas volcánicas (conos, coneletes escoriáceos, hornitos, cráteres, coladas...) en muy buen estado de conservación.

Partiendo de la posibilidad de que Montañas Negras sean fruto del paroxismo visto por Colón en 1492, el objetivo del trabajo es el análisis de sus elementos morfológicos para determinar en qué medida, éstos se asemejan, o no, a los rasgos morfovolcánicos de las erupciones históricas de Tenerife.

\section{APROXIMACIÓN DOCUMENTAL.}

En el Diario de a bordo de Cristobal el Colón en su primer viaje a las Américas, se cita una erupción en 1492: "Jueves 9 de Agosto... Después tornó el almirante a Canarias, y adobaron muy bien La Pinta con mucho trabajo y diligencias del almirante, de Martín Alonso y de los demás; y al cabo vinieron a La Gomera. Vieron salir gran fuego de la sierra de la isla de Tenerife, que es muy alta en gran manera.." (COLÓN, 1985: 42), "...Pero como después de la salida del carabelón tardó mucho en saber noticias, el Almirante resolvió a 23 de agosto volver con 
sus dos naves a la Gran Canaria: y así partiendo al día siguiente, encontró en el camino al carabelón, que no había podido llegar a la Gran Canaria, por serle el viento muy contrario. Recogió al hombre que lo guiaba, y pasó aquella noche cerca de Tenerife., de cuya montaña se veían salir grandísimas llamas; de lo que maravillándose su gente, les dio a entender el fundamento y la causa de tal fuego, comprobándolo todo con el ejemplo del Etna en Sicilia y de otros muchos montes donde se veía lo mismo..." (CIORANESCU, 1978: 67). Aunque la erupción parece contar con fecha perfectamente definida, su localización es aun incierta. Los datos que se poseen se reducen a la constatación del fenómeno eruptivo sin la descripción de los caracteres de la misma o la sucesión de las distintas fases de actividad.

De este modo, C. Romero indica que con cierta seguridad se podía establecer una fecha aproximada del fenómeno, 24 ó 25 de agosto de 1492, siguiendo los datos proporcionados por Fernando Colón (ROMERO, 1991: 38). Pero respecto a la localización espacial no todos los autores muestran unanimidad. Así, en 1964, Bravo y Coello señalan que Montaña Reventada, localizada en las faldas de Pico Viejo a unos 2.100 metros de altitud, era la erupción vista por Colón (BRAVO ET AL, 1980: 48). En 1991, Romero, al analizar las erupciones históricas de Tenerife, señala que M. Reventada presenta rasgos morfológicos y geoquímicos que difieren mucho de los paroxismos de edad histórica, no sólo de Tenerife sino del Archipiélago (ROMERO, 1991: 38). Corroboran esta idea Soler y Carracedo con las dataciones paleomagnéticas que sitúan la construcción de este aparato entreel año 1000 y 1300 (SOLER et al, 1986: 34).

Teniendo en cuenta la derrota seguida por el descubridor hacia la Gomera, con una visión general de la vertiente sur, sureste y suroeste de Tenerife, y que es en este último sector donde se encuentran restos de manifestaciones muy recientes que se encuadran en la diagonal Teide-Teno, permiten señalar que cualquiera de los aparatos volcánicos recientes ubicados en este ámbito (Montañas Negras, Montaña de Abeque, Boca Cangrejo etc) podrían corresponder a la erupción que Colón apunta en su diario. De este modo, Montañas Negras podría constituir un conjunto eruptivo generado durante una erupción histórica. Independientemente de si lo es o no, es destacable el hecho de que son volcanes de edad muy reciente.

\section{PARÁMETROS QUE DETERMINAN LOS RASGOS MORFOLÓ- GICOS.}

Los rasgos morfológicos de cada aparato volcánico depende de la combinación de múltiples parámetros: naturaleza y composición química del magma, tipo y disposición de la fractura, estilo y evolución dinámica, intensidad del paroxismo, acción del viento, rasgos topográficos del área de asentamiento de conos y coladas, duración de la erupción... (ROMERO, 1990: 179-180 y ROMERO, 1992: 5859). A pesar de que la morfología de los conos depende de la combinación de todos los parámetros antes mencionados, la importancia relativa de cada uno de ellos no es la misma.

Partiendo de que los edificios volcánicos son fruto de erupciones de tipo basáltico, que la topografía previa, suavemente inclinada, presenta desniveles muy 
poco acusados y que el viento, con una dirección dominante N-NE durante el paroxismo, no introdujo modificaciones considerables; hace pensar que dos son los factores que parecen haber jugado un papel esencial en la configuración de los rasgos morfológicos finales del conjunto eruptivo de Montañas Negras; por un lado, el tipo de fracturación y por otro la dinámica eruptiva.

Al no poseer documentación para ninguno de los volcanes que nos relaten aspectos de su formación, el estudio morfológico nos puede ayudar a solventar este problema. Ya que el sistema de fracturación es uno de los principales factores que intervienen en la morfología final de los aparatos volcánicos al condicionar su disposición espacial, y el comportamiento eruptivo y la evolución dinámica nos aporta información de los rasgos geomorfológicos de los conjuntos volcánicos resultantes de una erupción (conos, cráteres y coladas). Pero estableciéndose con mayor facilidad y fiabilidad a partir de los datos obtenidos de los aparatos volcánicos -conos y cráteres- que de los aportados por las corrientes lávicas (ROMERO, 1991: 252), puesto que éstas sólo nos dan información de las fases efusivas y de los aumentos y disminuciones de caudal en el caso de que se desborden las morrenas laterales, mientras que los edificios volcánicos aportan datos de las etapas efusivas y explosivas, indicándonos el comportamiento eruptivo de los mismos, sus rotaciones en las fases lávicas, como en la erupción del Teneguía, las diferentes etapas explosivas reflejadas en los repechos estructurales del interior de los cráteres o en las escorias que sueldan los sectores cimeros de los conos, etc.

\subsection{El sistema de fracturación como factor condicionante de la morfolo- gía de los edificios volcánicos.}

El conjunto eruptivo de M. Negras se desarrolla a partir de varias fisuras de igual rumbo -NW-SE-. La existencia de varias hendiduras de este tipo suelen corresponder a una grieta volcánica descompuesta en tramos diferenciados y desplazados (ROMERO, 1991: 121). Las dos fracturas, aunque presentan rumbos similares, están ligeramente desplazadas, de este modo, la que incluye los conos número cuatro, dos y $\mathrm{M}$. Aguda, con una longitud de 450 metros, posee una dirección NW-SE, y la que engloba los conos cinco, tres y cuatro de unos $400 \mathrm{~m}$. de largo, tiene una dirección WNW-ESE.

Fruto de este tipo de fracturación se construyen edificios fisurales que en nuestro caso no muestran continuidad morfológica a lo largo de toda la fractura por dos razones fundamentales: una, la esencial, es que la actividad se ha concentrado en ciertos puntos de la misma; dos, que ésta se descompone en tramos no muy diferenciados pero si lo suficientes como para dejar desconectados espacialmente el cono dos del tres y cinco. A su vez, los tramos activos aparecen unidos por el cruce de las dos fracturas en el edificio número cuatro, que es precisamente el que presenta mayores dimensiones y una evolución dinámica más compleja.

El resultado es un conjunto de aparatos alineados que se forman a partir de la individualización y yuxtaposición de fracturas que dan lugar a sectores con distinto grado de potencialidad eruptiva, siendo máxima en los extremos y cruce de las líneas estructurales. 


\subsection{Los rasgos morfológicos como indicadores de la dinámica eruptiva.}

A través del análisis de los rasgos morfológicos podemos intuir los estilos eruptivos, aunque la relación es a la inversa, es decir, la dinámica eruptiva condiciona, al igual que el sistema de fracturación, de forma directa la morfología final de los aparatos volcánicos.

Por la moderada explosividad deducida de la morfología de los edificios, la dinámica la podríamos encuadrar dentro del tipo estromboliano definido por Lacroix, pero si tenemos en cuenta que los materiales arrojados por este tipo de erupciones existe una proporción semejante entre coladas de lava y materiales piroclásticos (DERRAUX, 1983: 320), y que en estos volcanes, de forma general, predominan los materiales efusivos frente a los explosivos, el estilo eruptivo de éstos no es estromboliano sensu estricto, esto no quiere decir que tengamos que encuadrarlos en algunos de los restantes estilos definidos por Mercalli y Lacroix. Por lo tanto, estamos ante erupciones cuyo dinamismo es intermedio, entre el estromboliano y hawaiano, es un dinamismo de tipo mixto, que puede ser clasificado como estrombohawaiano o estromboefusivo.

Durante el período activo, los estilos eruptivos se han sucedido y yuxtapuesto, de tal manera que la morfología final de este conjunto volcánico está en relación con la evolución dinámica espaciotemporal registrada durante las erupciones, así, en los extremos de las fracturas predominan los estilos hawaianos y estromboefuisvos manifestado a través de la presencia de pequeños conos escoriáceos que derraman una colada, en proporción, mayor que ellos mismos, mientras que en el tramo central de las mismas, la presencia de embudos explosivos y algunos campos de lapillis, nos da una idea de un comportamiento más explosivo.

\section{RASGOS MORFOLÓGICOS DE LOS VOLCANES DE MON- TAÑAS NEGRAS.}

Estas erupciones se originaron en torno a dos fisuras de rumbo NW-SE y WNW-ESE, en ellas son frecuentes las construcciones volcánicas de diferentes dimensiones entre las que se encuentran edificios abiertos en herradura, cónicos y anulares. Los cráteres también presentan variedad morfológica, desde los embudos explosivos anulares a los abiertos en herradura típica, de vértices prolongados e incluso incipientes. Todos los edificios y centros eruptivos presentan una morfología interna diferenciada, pero organizada en el espacio siguiendo las pautas determinadas por la línea estructural NW-SE.

\subsection{Rasgos morfoestructurales de los aparatos volcánicos.}

A lo largo de las líneas eruptivas es posible diferenciar, por su disposición espacial y morfológica, varios sectores imbricados y yuxtapuestos a veces, e individualizados otras, pero todos, en general, le otorgan al conjunto una disposición de carácter lineal.

La concentración de la actividad en puntos concretos de las distintas fracturas nos permite establecer sectores individualizados:

- Hacia el sureste encontramos un pequeño cono escoriáceo, con cráter en 
herradura, de marcado carácter efusivo e imbricado al sector septentrional de Montaña Aguda.

- En el tramo central encontramos dos aparatos de diferentes proporciones, el localizado hacia el sureste presenta un embudo de explosión unido a un cráter rebajado que derramó colada. Al noroeste tenemos un edificio anular de carácter explosivo. De manera general, la actividad explosiva es predominante, así lo confirman los campos de lapillis y los embudos de explosión.

- Al noroeste tenemos un cono compuesto por materiales de proyección aérea, cementado por escorias en su cima y con cráter en herradura en el que el carácter efusivo también es más importante que el explosivo.

\subsubsection{Sector suroriental.}

Morfológicamente en este tramo de la fisura se construyó un edificio escoriáceo, disimétrico, de carácter lineal y adosado a Montaña Aguda en sus bordes norte y noreste. Por este motivo el aparato es disimétrico, ya que a la mayor facilidad de acumularse las escorias y lapillis en el cráter de Montaña Aguda, se le une, de un lado, la dirección dominante del viento durante la erupción, y de otro, relacionado con lo anterior, parte del dorso norte del edificio está cubierto por los lapillis procedentes del cono número cuatro, esto permite un mayor desarrollo altitudinal y unas pendientes más acusadas de estos flancos frente al resto. La pared este del cono, compuesta por escorias y lapillis en proporciones similares, está solapada al tramo inferior central del cráter de Montaña Aguda, mientras que el flanco oeste no existe, puesto que es un cono en herradura alargado y abierto hacia el mismo.

El cráter de este edificio presenta un marcado carácter fisural. En su tramo superior se vislumbra una incipiente boca explosiva semicircular compuesta por escorias soldadas en su borde sur y recubierto en el resto por taludes escoriáceos. En su tramo medio-bajo, tenemos un salidero que derrama colada, en la que es muy difícil apreciar si se produce desbordamiento, puesto que el cráter en este sector está a la misma altura que los flancos norte y sur, a su vez, el inicio del derrame está tapizado por un campo de lapillis, lo que dificulta aún más precisar si la colada rompió el flanco oeste, o que nunca existió. La presencia de la boca incipiente junto con el marcado carácter lineal, nos da una idea de una dinámica mixta, en la que explosiones y efusiones funcionaron coetáneamente.

\subsubsection{Sector central.}

Este tramo no constituye una unidad volcánica uniforme y tampoco es resultado de un comportamiento eruptivo homogéneo a lo largo de la fisura y durante el período activo, de ello dan muestras la diversidad de formas.

Morfológicamente diferenciamos dos aparatos volcánicos separados por una corriente lávica. Hacia el noroeste tenemos un conelete explosivo en forma de cono anular compuesto por escorias y lapillis. Su cráter es el típico embudo explosivo formado por escorias aplastadas, soldadas y adaptadas a la topografía previa en las que se observan grietas de retracción fruto del enfriamiento y solidificación posteruptivos. En las paredes internas del mismo existen tres repechos estructurales fruto de diversas fases de actividad relacionados con los distintos momentos eruptivos de carácter explosivo, incluso, por el recubrimiento del material que los tapiza, posteriormente pudo haber funcionado como un charco de la- 
va. El fondo del cráter está recubierto por escorias y piroclastos que durante la propia fase explosiva se depositaban en el borde del embudo y caían por gravedad, o bien lo hacían directamente debido a las proyecciones verticales, o por los procesos de dinámica de vertiente posteruptivos. De estos rasgos morfológicos es fácil deducir la dinámica fundamentalmente explosiva que originó el aparato.

Hacia el sureste tenemos un gran cono volcánico con dos bocas cratéricas de desigual dimensiones, morfología y dinamismo. Es un aparato formado por escorias heterométricas, jirones de lava y lapillis que conforman un edificio disimétrico, más elevado en su flanco norte-noreste como consecuencia de la propia dinámica eruptiva y de las constancia del viento en esa dirección durante el paroxismo. Hacia el sur, el edificio conecta con una montaña de lapillis compuesta por escorias efusivas y bombas volcánicas dispersas.

De este aparato lo más destacable son sus dos grandes cráteres -grandes en relación con el de los demás edificios-, hacia el E-SE existe un embudo explosivo circular, con una profundidad de unos 12 metros y con uno de sus flancos parcialmente destruido por la apertura de la segunda boca hacia el oeste. En las paredes internas del embudo existen dos rellanos estructurales constituidos por escorias soldadas y lapillis, mientras que el fondo del cráter está tapizado por escorias de gran tamaño fruto de la selección gradual de las mismas por dinámica eruptiva y gravitatoria. El que el embudo tenga mayores dimensiones y presente dos repechos estructurales frente a los tres del embudo explosivo anterior, nos da una idea de una dinámica explosiva más sencilla pero mucho más violenta.

Hacia el noroeste encontramos la otra boca, es un cráter rebajado y abierto en dicha dirección y dinámicamente más complejo que el anterior pero menos profundo, diez metros,. Su origen se debe por un lado, a la proyección oblicua de materiales debido a la inclinación del conducto emisivo y ayudados por la intensidad del viento en esa dirección, y por otro, la emisión de lavas a favor de la pendiente desbordando el cráter e impidiendo la construcción del edificio en ese sector. En el interior de las paredes cratéricas existen taludes escoriáceos que conectan con el fondo del cráter, tapizado por una superficie lávica heterogénea y caótica que presenta pequeñas cavidades intravolcánicas fruto de su propia morfología. En el interior del mismo, se observa un gran nivel de rellano estructural compuesto por escorias muy consistentes que coincide con el nivel altitudinal en el que el embudo explosivo localizado hacia el sureste está destruido, siendo fruto del dinamismo que lo formó. Dinámicamente es más complejo porque en él apreciamos formas propias de momentos explosivos, repechos estructurales, y también de fases efusivas como la corriente lávica hacia el noroeste.

\subsubsection{Sector noroccidental.}

Este sector se encuentra separado espacialmente del resto de las construcciones de esta misma alineación volcánica, la interrupción local de la fisura eruptiva es de unos 23-25 metros aproximadamente como consecuencia de la concentración de la actividad en el extremo noroccidental de la fractura WNW-ESE.

Morfológicamente existe un edificio cónico de unos doce metros de altura formado por lapillis y escorias sueltas en las cotas medio bajas, y cementadas, en la parte superior del aparato, fruto de la combinación de una dinámica explosiva poco intensa que fragmenta el magma, que incandescente, cae en los flancos del cono, se solidifica y se compacta. Alrededor del conjunto existe un campo de lapillis con algunas bombas volcánicas aisladas, y en el que se aprecian indicios de 
pillis con algunas bombas volcánicas aisladas, y en el que se aprecian indicios de fenómenos de escorrentía con la presencia de reguerrillos.

El cráter, en herradura, está compuesto por escorias soldadas, en unos sectores, y lapillis, que debido a los procesos de dinámica de vertiente dan lugar a taludes escoriáceos que recubren y tapizan las paredes internas y el fondo del mismo.

Dinámicamente, este conjunto, presenta rasgos propios de momentos efusivos y explosivos que se sucedieron en el tiempo de forma coetánea, pero debido a que los materiales de proyección aérea recubren parcialmente la colada procedente del cono cuatro y la suya propia, nos está indicando que el paroxismo culminó con una fase explosiva.

\subsection{Rasgos morfoestructurales de las corrientes lávicas.}

De la combinación de los factores intrínsecos (quimismo del magma, cualidades físicas del fundido (temperatura, fluidez, contenido en gas...) y la dinámica eruptiva) y extrínsecos (topografía, ámbito de desarrollo...) depende la morfología superficial y la disposición espacial de las coladas. El carácter básico de los magmas, la dinámica estromboefusiva y una pendiente moderada que condiciona el derrame de las corrientes lávicas a favor de la misma, junto con la disposición lineal del conjunto que favorece el recorrido del fluido a ambos lados de la fractu$\mathrm{ra}$, determina que la planta de las coladas sea en corbata arrugada, partiendo de un nudo en las bocas emisivas para progresivamente incrementar su perfil transversal y terminar estrechándose en sus frentes.

La morfología superficial de las coladas, generalmente es $a a$, cuyo rasgo esencial es una cobertera rota, áspera, rugosa, constituida por fragmentos heterométricos, móviles y de textura escoriácea. Las formas superficiales son pedazos irregurales de bordes quebrados y vesiculados. Dentro de la gama de las $a a$ se aprecian diversas morfologías superficiales de transición de coladas pahoehoe con grietas de retracción paralelas entre sí hacia $a a$. Los frentes de las coladas lo constituyen un talud de derrubios escoriáceos cuyo origen se debe, fundamentalmente, al modo en que la colada avanzó durante su derrame, donde la parte móvil superficial cae al pie del frente de progresión, formando un talud móvil que la colada cubre a medida que prosigue el desplazamiento. En definitiva, una superficie cubierta por material fragmentado y suelto.

Dentro de las corrientes lávicas diferenciamos formas mayores y menores. En las primeras destacan los muros laterales de enfriamiento, los canales de derrame subaéreos y los hornitos. Los dos primeros están íntimamente relacionados y su génesis responde a un proceso de enfriamiento diferencial del cauce con respecto a los laterales, es decir, los muros se enfrían y por el interior del mismo la lava continua discurriendo. En algunos casos, estos canales presentan niveles inferiores fruto de derrames tardíos que tienden a fluir por los sectores que presentan mayor facilidad, y donde la lava no es suficiente como para desbordar al antiguo canal. Los hornitos o bocas incipientes presentan en su vértice una apertura circular por la cual escapan los gases. Son construcciones con o sin raíz que se forman sobre la colada por el apilamiento de paquetes de lava fluida y proyectada fruto de una breve y tenue actividad limitada a la expulsión de gases. En las formas menores predominan los bloques escoriáceos y los erráticos; los primeros se loca- 
lizan en el inicio de los derrames lávicos y próximos a los sectores terminales de las bocas emisivas, son paquetes de lava de gran potencia, muy irregulares y asociados a magmas más viscosos. Los segundos, constituyen pedazos del edificio volcánico que han sido arrancados y desplazados por el flujo de lava, o bien son resultado del resquebrajamiento de la propia corriente en su recorrido.

Internamente las coladas presentan una estructura que no es homogénea, compuesta de tres sectores diferenciados. Un nivel superior escoriáceo fruto de la desgasificación, enfriamiento y movimiento de la colada. Un nivel central de roca masiva de textura más o menos vacuolar según las cualidades físicas del magma que la ha originado y en las que podemos apreciar la incipiente estructuración columnar, propia de los magmas básicos y debido a procesos de retracción de la lava durante su enfriamiento, ya que la escasa potencia de los derrames impide la perfecta formación de la misma. Y en la parte inferior aparece un estrato escoriáceo basal debido al rápido enfriamiento de la corriente en contacto con el suelo previo.

\section{CONCLUSIONES.}

Antes de llevar a cabo el análisis morfológico de Montañas Negras, éstas contaban con dos aspectos muy importantes que nos permitía pensar que podían ser fruto de la erupción de 1492: presentar un aspecto muy reciente y estar localizadas al suroeste de Tenerife en el tramo cimero de la isla.

Los volcanes de Montañas Negras son un conjunto eruptivo de carácter basáltico, pertenecientes a las series recientes básicas, al igual que el resto de las erupciones históricas de Tenerife. Constituyen un episodio monogénico que utiliza varias fisuras de igual rumbo NW-SE, la misma directriz que explotan los conjuntos volcánicos de edad histórica, Garachico 1706 y Chinyero 1909, localizados en el mismo sector que Montañas Negras. Su comportamiento y dinámica eruptiva es de tipo mixto, asemejándose con las erupciones históricas de baja o nula explosividad, o con los paroxismos de explosividad moderada.

Por lo tanto, los rasgos morfovolcánicos de los conjuntos de edad histórica de la Isla y Montañas Negras responden a esquemas estructurales y comportamientos dinámicos de escasa complejidad, de este modo, las formas resultantes de unos y de otros se encuadran en las categorías morfológicas de gran sencillez.

De este modo, a los aspectos señalados anteriormente, hay que añadirle la similitud morfológica de este conjunto con los históricos, lo que nos corrobora aún más la posibilidad de que la erupción de 1492 observada por Colón sea la que aquí se ha tratado.

\section{BIBLIOGRAFÍA.}

- BRAVO, T. Y CoEllo, J. (1980); «Sabino Berthelot y su influencia en la geología de Canarias». Homenaje a Sabino Berthelot en el centenario de su fallecimiento 1880-1980. Instituto de Estudios Canarios, La Laguna.

- Colón, C. (1985); Diario de a bordo. Ed. Generales Anaya, Madrid.

- Cioranescu, A. (1978); Colón y Canarias. Aula de la cultura, S/C de Tenerife. 
- DeRrAUX, M. (1984); Geomorfología. Ariel, Barcelona.

- DóNIZ PÁEZ, J., ARMAS AYALA,V. Y RoMERo RuIZ, C. (1999): «Organización espacial de la actividad eruptiva en la dorsal volcánica de Pedro Gil (Tenerife, Islas Canarias)». El territorio y su imagen, vol, I, Actas del XVI Congreso de Geògrafos Españoles. Universidad de Málaga, Málaga. pp. 81-90.

- ROMERO, C. (ED.) (1990); Jornadas de campo sobre geomorfología volcánica. Monografía $\mathrm{n}^{\circ}$ 5, SEG, Zaragoza.

- ROMERO, C. (1991); Las manifestaciones volcánicas históricas del Archipiélago Canario. Gobierno Autónomo de Canarias, Consejería de Política Territorial, $\mathrm{S} / \mathrm{C}$ de Tenerife.

- ROMERO, C. (1992); Estudio geomorfológico de los volcanes históricos de Tenerife. ACT. Aula de Cultura, Cabildo de Tenerife, S/C de Tenerife.

- SOLER, V. Y CARRACEDO, J. (1986); «Aplicación de técnicas paleomagnéticas de corto período a la datación del volcanismo subhistórico de la isla de Tenerife». Geogaceta, $\mathrm{n}^{\mathrm{o}}$ 1, pp. 33-35. 
MAPA 1. Localización del conjunto de Montañas Negras y su relación espacial con el volcanismo histórico de Tenerife.

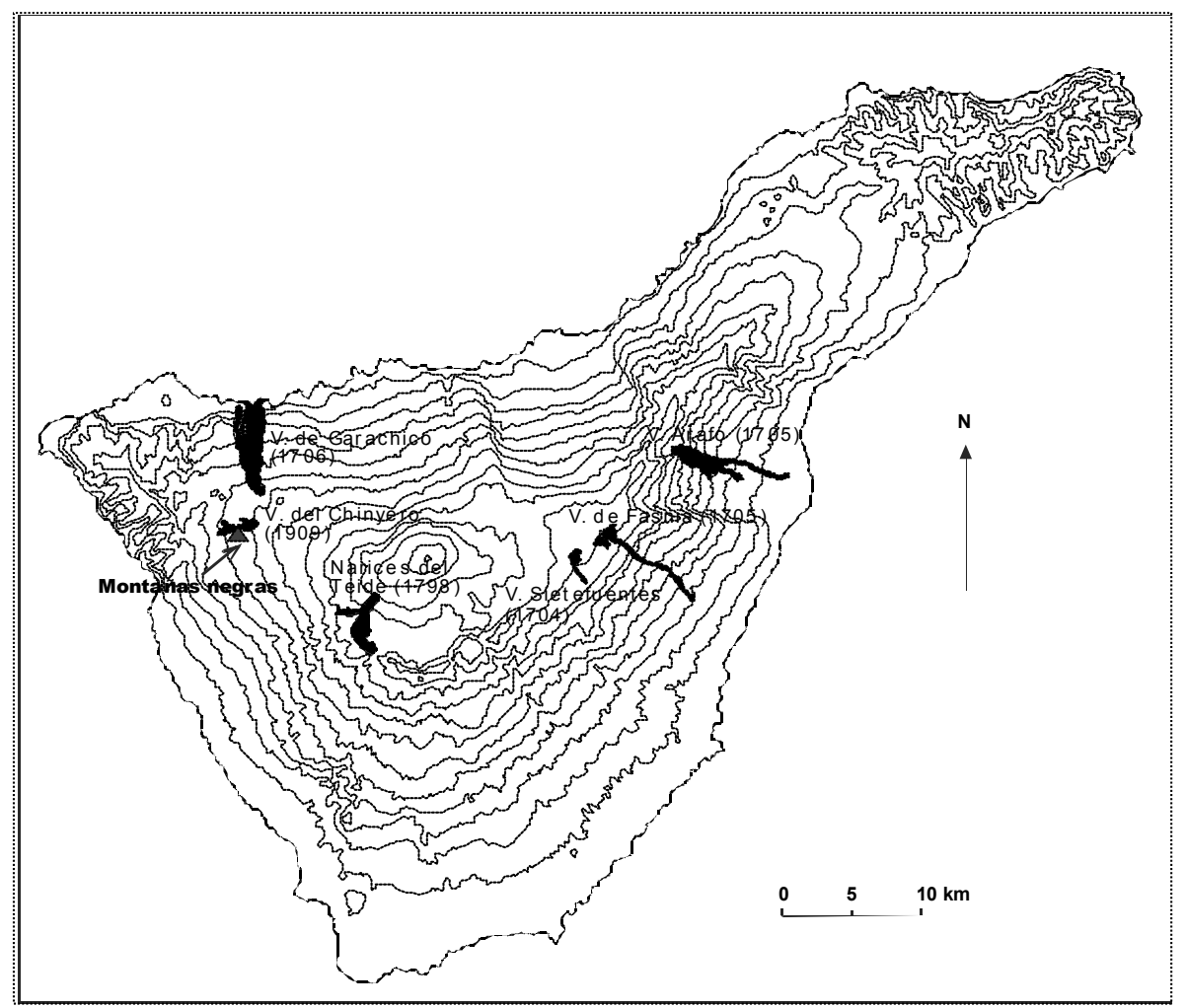

CUADRO 1: Principales rasgos morfométricos de las coladas.

\begin{tabular}{lcccl}
\hline $\begin{array}{l}\text { Colada } \\
\text { lávica. }\end{array}$ & $\begin{array}{l}\text { Desarrollo } \\
\text { longitudinal }\end{array}$ & $\begin{array}{l}\text { Desarrollo } \\
\text { transversal }\end{array}$ & $\begin{array}{l}\text { Morfología } \\
\text { superficial. }\end{array}$ & $\begin{array}{l}\text { Principales elementos morfológi- } \\
\text { cos. }\end{array}$ \\
\hline A & $400 \mathrm{~m}$. & $100 \mathrm{~m}$. & aa y losas & $\begin{array}{l}\text { Bloques escoriáceos y muros latera- } \\
\text { les. }\end{array}$ \\
$\mathrm{B}$ & $500 \mathrm{~m}$. & $100 \mathrm{~m}$. & aa & $\begin{array}{l}\text { Islotes y bloques erráticos, escoriá- } \\
\text { ceos y canal lávico. } \\
\text { Canales lávicos }\end{array}$ \\
$\mathrm{C}$ & $350 \mathrm{~m}$. & $105 \mathrm{~m}$. & aa &
\end{tabular}


Croquis morfológico del conjunto eruptivo de Montañas Negras.

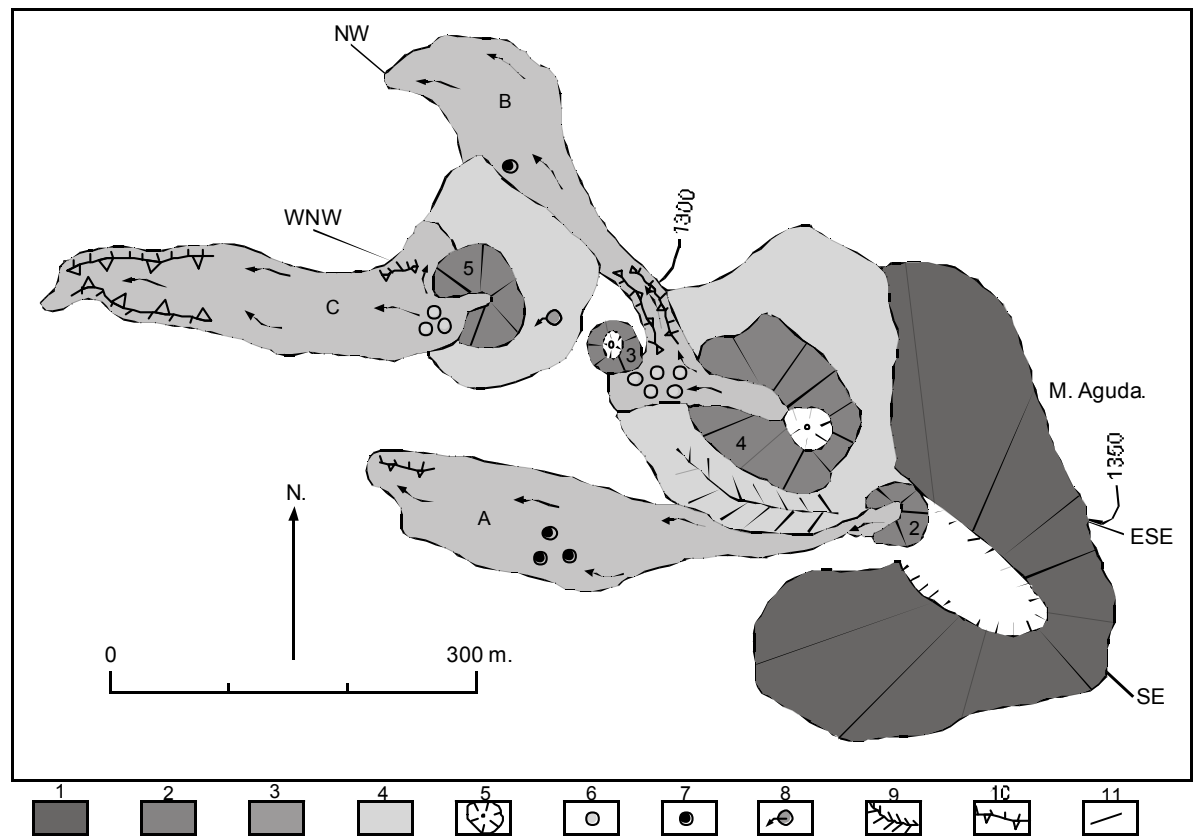

1, Volcán de una erupción anterior. 2, Volcanes de Montañas Negras. 3, Lavas. 4, Campos de lapillis. 5, Embudos de explosión. 6, Bloques escoriáceos. 7, Bloques erráticos. 8, Hornito. 9, Montaña de piroclastos. 10, Muros y canales lávicos. 11, Pautas estructurales. 